Theories \& Applications, the International Edition

Printed Version: (ISSN 2090-5262)

Online Version: (ISSN 2090-5270)

November 2013, Volume 3, No. 3 Pages (154 - 162)

\title{
Impacts of Concurrent Strength and Endurance Training on Vo2 Max and Certain Physical Variable among Young Tennis Players in Kuwait
}

\author{
Mubarak AbdullReda Ali Reda*, Ahmed Ali Alfailakawi**
}

\begin{abstract}
Concurrent training is the idea of combining both endurance training and weight training into one program. The purpose of this study is to investigate the effects of concurrent training on cardiopulmonary response, power and endurance among young tennis players. Thirty young tennis players who are playing in Khazma Club and all registered in the Kuwaiti Union of Tennis, and their ages are rounding between 14.5 yrs to 15.5 yrs divided into three experimental groups concurrent $(C G, n=10)$, resistance $(R G, n=10)$, and endurance training group $(E G, n=10)$. Each group trained three times a week for eight weeks, all types of training in the same session. Parameters assessed the high, weight, and power, strength, training age, VO2 max (Astrand Treadmill Test was used to determine the VO2 max) and training experience. All participants were free of any disorders known to affect performance, such as bone fractures, Osterporosis, diabetes and cardiovascular disease or recent surgery. The participants did not report use off any antiseizure drugs, alcohol and cartoon consumption, neither smoking cigarette. And all participants were fully informed about the aims of the study, and gave their voluntary consent before participation. The measurement procedures were in agreement with the ethical human experimentation. Participants completed 8-10 resistance-training exercises first, and then completed their hour of training by walking/jogging/running for up to 30 minutes on a treadmill at a prescribed target heart rate. Dynamometer instrument was measured the strength of leg and back. The results revealed that significant differences in Grip strength between Strength group and concurrent group for concurrent group. Taking into consideration that significant difference in Grip strength between endurance group and concurrent group for concurrent group, where significant differences in VO2 max between endurance group for concurrent group are calculated. There was a significant difference in LS between strength group and endurance group for strength group. No significant differences in LS between strength group and concurrent group were calculated and significant difference in LS between endurance group and concurrent group for concurrent group were calculated as well. While significant differences in BS between strength group and endurance group for strength group were calculated. No significant differences in BS between endurance group and concurrent group, and significant differences in BS between endurance group and concurrent group for concurrent group were calculated. In conclusion, the study has shown that eight weeks of concurrent strength and endurance training has beneficial effects on musculoskeletal power and VO2 max.
\end{abstract}

Keywords: VO2 Max, strength, power

\footnotetext{
* Professor Association at College of Basic Education -Department of Physical Education and Sport-The Public Authority For Applied Education and Training-State of Kuwait

** Professor Association at College of Basic Education -Department of Physical Education and Sport-The Public Authority For Applied Education and Training-State of Kuwait
} 


\section{Introduction}

$\mathrm{I}^{\mathrm{m}}$ mproving tennis performance is the goal of every tennis scientist, coach, and athlete. The practicality of this information should be applied when designing training programs for higher lever tennis players. Thirty young tennis players who are playing in Khazma Club and all registered in the Kuwaiti Union of Tennis, and their ages are rounding between $14.5 \mathrm{yrs}$ to 15.5 yrs divided into three experimental groups concurrent $(\mathrm{CG}, \mathrm{n}=10)$, resistance $(\mathrm{RG}, \mathrm{n}=10)$, and endurance training group $(\mathrm{EG}, \mathrm{n}=10)$. All subjects signed written consent forms to participate in both the laboratory and field test protocols. The consent form,approved by the Department of Physical Education and Sport at College of Basic EducationAnd each group trained three times a week for eight weeks, all types of training in the same session. Parameters assessed the high, weight, and power, strength, training age, VO2 max (Astrand Treadmill Test was used to determine the VO2 max) and training experience. Age, sex, style of play, physical components technical components, tactical components, and psychological components will all determine the success of the tennis athlete. Effective planning and training programs will help in designing a safe, effective, and productive program design to help optimize performance. That dependent on optimum muscle function to generate the forces required in tennis and to protect against the loads applied to the body as a result of tennis playing. Strength is the ability to generate a force or protect against a load power is the ability to do that quickly, and endurance is the ability to do that over extended times. Muscle balance allows maximum joint protection and smooth motion extended times. Muscle balance allows maximum joint protection and smooth motion of joints. Muscles may develop alterations due to lack of conditioning, wrong emphasis in training, fatigue, injury, or thixotropy.

\section{Literature Review}

Tennis demands also the anaerobic and aerobic abilities, which necessitates the simultaneous incorporation of training strategies designed to develop both systems. Many tennis players train with little or no emphasis on power development or in a manner secondary to aerobic development. Such practices are illogical, as indicated by the physiological demands of tennis (Malina et al., 2005). Additionally, even if tennis were predominantly aerobic activity, anaerobic training in far less effective when employed secondary to aerobic development, while aerobic systems do not seem to suffer interference from anaerobic development processes. Contrary to popular belief, varying resistance exercise programs have been shown to enhance performance in highly aerobic activities such as marathon running (Jung, 2003; Hopkins, 2009).

Few studies have addressed the performance needs ofcompetitive tennis players.Dramatic changes in physiological attributes typically occurat the age of about $12-15$ years, but there is a large inter-subject variability in the timing of maturation. Considerable factors such as the age of peak height velocity are known to influence to agreat extent the physical factors. However, it is still unclearhow the stage of maturation influences the acquisition of the specific/technical skills (Bencke, et al., 2002). Therefore, the influence ofphysical abilities on tennis play may be more apparent duringthis time of development due to dramatic increase instrength, size, and endurance. Knowledge of the relationship between various physical attributes such as speed,explosive power, leg stiffness, muscular strength of upper and lower limbs and the ranking of adolescent tennis playerscould assist in determining the relative importance of suchmeasures and providing optimal training programs. Inaddition, little is known regarding the nature of the relationships between such physical variables in this age group of tennis players (Girard and P.Millet, 2009).

The combination of resistance training (RT) and endurance training (ET) is frequently used in athletic. These terms are used to characterize the method whereby aerobic and strength training exercises are performed in the same training session (Bell, et al., 2000; Dantas, el al. 2008). That strategy was chosen because energy expenditure could be maximized both during and after the training through increased oxygen consumptions after exercise no.18, while some 
authors mention concurrent training in their publications (Davis, et al., 2008).

The specificity of training principle states that the nature of tissue adaption after training is dependent on the specific type of training participated (Nieman, 2003). As a corollary to this principle, combining two types of training (e.g., resistance and endurance training) may interfere with the training response induced by either type of training alone. Reasonable physiologic and metabolic evidence exists to support this principle.

Designing and implementing training for tennis requires a solid understanding of many physiological variables critical to optimal performance. Tennis requires short explosive bursts of energy repeated dozen, if not hundreds of times per match or practice session. Tennis unlike many other sports does not have time limits on matches. This can result in matches lasting less than one hour or as long as five hours (in five-set matches). This variability requires successful tennis athletes to be highly trained both anaerobically for performance, and aerobically, to aid in recovery during and after a play (Hopkins, 2009).

Oxygen uptake (VO2) at maximal exercise is considered the best index of aerobic capacity and cardio-respiratory function. Maximal VO2 is defined as the point at which no further increase in measured $\mathrm{VO} 2$ occurs and a plateau is reached, despite an increase in work rate during graded exercise testing.

Strength and endurance training regimes represent and induce distinctly different adaptive responses when performed individually. Typically, strength training programs involved large muscle group activation of high-resistance and low repetition exercises to increase the force output ability of skeletal muscle (Girard, et al., 2006). In contrast, endurance training programs utilize low resistance, high repetition exercises such as running or cycling to increase maximum $\mathrm{O} 2$ uptake (VO2 Max). Accordingly, the adaptive responses in skeletal muscle to strength and endurance training are different and sometimes opposite (Girard and P.Millet, 2009). Therefore, the purpose of this investigation was to examine the effects of concurrent training on cardiopulmonary response, power and endurance among young tennis players.

\section{Material and methods}

\section{Experimental approach to the problem}

Three experimental groups performed a pre and post training designed intervention in which (VO2 Max). Heart rate during the effort (HR), and the physical variables contains grip strength (GS), leg strength (GS), leg strength (LS), back strength (BS), Standing Long Jump test (SLJ) and strength endurance for leg and arms (SEL) (SEA).

The experimental first group of (10 young tennis players) trained one hour per day, and three times a week on resistance program for eight weeks. The second experimental group (10 young tennis players) trained one hour per three times a week on endurance training program on the treadmill for eight weeks. The third experimental group (10 young tennis players) trained one hour per three days a week on concurrent training program for eight weeks. While the three experimental groups completed the training programs to see whether this type of training modality would a positive or negative or no effect on (VO2Max), (HR), (GS),(LS),(BS),(SEL), and (SEA).

\section{Samples}

Thirty young tennis players divided into three equal experimental groups concurrent $(\mathrm{CG}$, $\mathrm{n}=10)$, resistance $(\mathrm{RG}, \mathrm{n}=10)$, and endurance training group $(\mathrm{EG}, \mathrm{n}=10)$. Each group trained three times a week for eight weeks, as all types of training in the same session were conducted. Parameters assessed the high, weight, and power, strength, training age, $\mathrm{VO} 2$ Max as Astrand Treadmill test was used to determine the VO2 Max and training experience. All participants were free of any disorders known to affect performance, such as bone feature, Osterporosis, diabetes and cardiovascular disease or recent surgery. The participants did not report use off any anti-seizure drugs, alcohol and cartoon consumption, neither smoking cigarette. And all participants were fully informed about the aims of the study, and gave their voluntary consent before participation. The 
measurement procedures were in agreement with the ethical human experimentation.

\section{Training Protocol}

The eight weeks in season training program consisted of resistance training (RT) and endurance training (ET), as follow:

\section{Testing Procedures}

Participants were assessed before and after eight week of concurrent training program, where all measurements were taken one week before and after training at the same time of day. Tests followed a general warm-up that consisted of running, calisthenics, and stretching.

\section{Astrand Teadmill Test (Att)}

There is undertaking instruments should be conducted throughout the test:

- Treadmill

- Stopwatch

- Assistant

This test requires the athlete to run as long as possible on a treadmill whose slope increments at times intervals, which are:

- The athlete warms up for 10 minutes.

- The assistance sets up the treadmill with a speed of $8.05 \mathrm{~km} / \mathrm{hr}(5 \mathrm{mph})$ and am incline of $0 \%$.

- The assistant gives the command "Go", starts the stopwatch and the athlete commences the test.

- The assistant, after 3 minutes into the test, adjusts the treadmill incline to $2.5 \%$ and then every 2 minutes thereafter increases the incline by $2.5 \%$.

- The assistant stops the stopwatch and records the time when the athlete is unable to continue.

From the total running time an estimate of the athlete's VO2 Max can be calculated as follows:

$$
\begin{gathered}
\text { VO2 Max mls } / \mathrm{kg} / \mathrm{min}=\left(\text { Time }^{*} \text { 1.44 }\right)+14.99 \\
\ldots . .(1)
\end{gathered}
$$

Where: Time is the recorded test time expressed in minutes and fractions of a minute.

\section{Push-Up Test}

A standard push up begins with the hands and toes touching the floor, the body, and legs in a straight line, feet slightly apart, the arms at shoulder width apart, extended and at a right angles to the body. Keeping the back and knees straight, the participant lowers the body to a predetermined point, to touch some other object, or until there is a 90-degree angle at the elbows, then returns back to the starting position with the arms extended. This action is repeated, and test continuous until exhaustion, or until they can do no more in rhythm or have reached the target number of push-ups.

\section{Dominate Grip Strength Test (Gs)}

The participant holds the dynamometer is the hand to be tested, with the arm at right angles and the elbow by the side of the body. The handle of the dynamometer is adjusted if required, which represents the base that should rest on first metacarpal (heel of palm), while the handle should rest on middle of four fingers. When ready the participants squeeze the dynamometer with maximum isomertic effort, which is maintained for about $5 \mathrm{sec}$. No other body movement is allowed. The participant should be strongly encouraged to give a maximum effort.

\section{Static Strenght Test (Ls) (Bs)}

A back dynamometer was used to measure the static leg strength. The participants stood on the dynamometer platform and crouched to the desired leg bend position, while strapped around the waist to the dynamometer. At a prescribed time they exerted a maximum force straight, head erect and chest high. Three trials were allowed to the subjects and the best score was taken. Participants had a rest between the trials.

\section{Standing Long Jump Test (Slj)}

The participant stands behind a line marked on the ground with feet slightly apart. A two foot take-off and landing is used, with swinging of the arms and bending of the knees to provide forward drive. The participant attempts to jump as far as possible, landing on both feet without falling backwards. Three attempts are allowed.

\section{Wall Sit Test (Wst)}

The participant stands comfortably with feet approximately shoulder with apart with his back against a smooth vertical wall. Slowly slide participant's back down the wall to assume a position with both his knees and hips at a 90 
angle. The training starts when one foot is lifted off the ground and is stopped when the subject cannot maintain the position and the foot is returned to the ground. After a period of rest, the other leg is tested. The total time is seconds that the position was held for each leg is recorded.

\section{Statistical Analysis}

All statistical analyses were calculated by the SPSS statistical package. The results are reported as means and standard deviations (SD). Differences between two groups were reported as mean differences + or $-95 \%$ confidence intervals (meandiff + or $-95 \%$ CI). Experimental test for independent samples was used to determine the differences in vision parameters between the two groups. The $\mathrm{p}<0.05$ was considered as statistically significant.

\section{Results}

The following table is representing the reading taking of participants' age, Anthropometric Characteristics and Training experience of all the three groups. As shown in Table 1. calculations the standard deviation is meant to be "Mean or SD".

Table (1)

Participants Groups Age, Anthropometric Characteristics and Training experience.

\begin{tabular}{|c|c|c|c|c|c|}
\hline Groups & $\mathrm{N}$ & Age [yrs] & Weight [kg] & Height [cm] & Training experience \\
\hline RG & 10 & $15.89+1.34$ & $62.47+4.3$ & $171.16+5.6$ & $5.00+1.2$ \\
\hline EG & 10 & $15.00+1.01$ & $60.35+4.4$ & $169.29+5.2$ & $4.94+1.6$ \\
\hline CG & 10 & $15.14+1.13$ & $63.04+5.2$ & $168.29+6.6$ & $5.03+0.9$ \\
\hline
\end{tabular}

Table 1.declares that the range of the young tennis participants players ages are varying from almost 15 years old to almost 16 years old, while their weights are varying from almost $63 \mathrm{~kg}$ to almost $60 \mathrm{~kg}$, and their heights are varying from almost $171 \mathrm{~cm}$ to almost $167 \mathrm{~cm}$. The training experience for each group of the training experience was varying from 5.03 to
4.94. There were no significant differences were observed in the anthropometric characteristics and Training Experience for the participants in the three groups. Figure 1, Figure 2, and Figure 3 represent the modulation for "Height", "Weight", "Age", and "Training Experience" of participants of each group.

Figure (1)

Modulation of Group RG Participants.

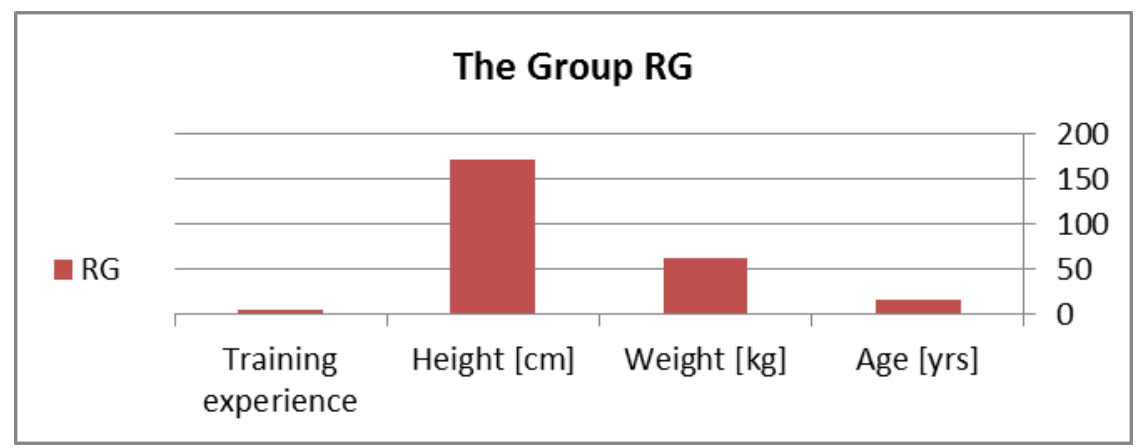

Figure (2)

Modulation of Group EG Participants.

\begin{tabular}{|lll|l|}
\hline \multicolumn{4}{c}{ The Group EG } \\
\hline \\
\hline
\end{tabular}


Figure (3)

Modulation of Group CG Participants.

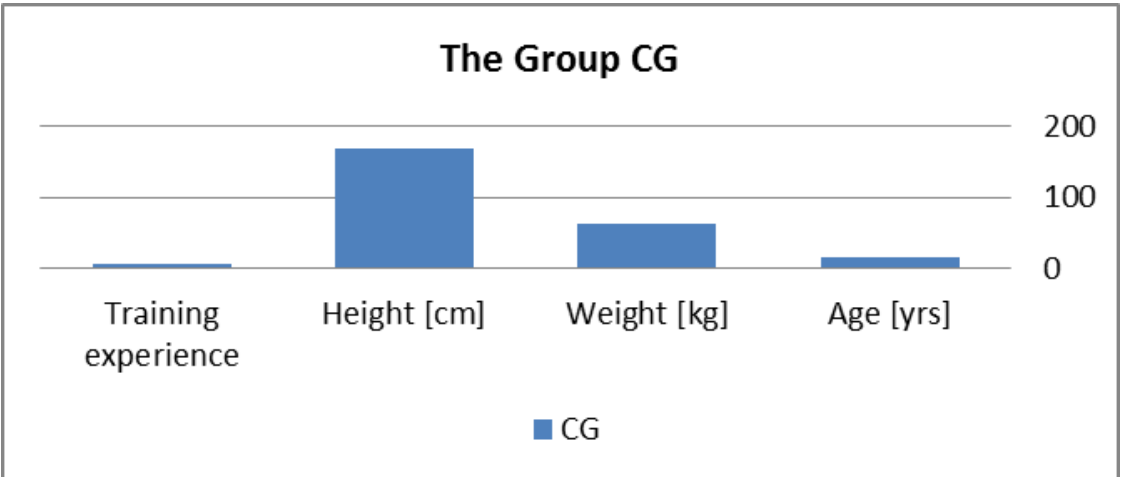

The following Table 2.toTable 8. illustrate the calculations of ANOVA test for VO2 Max and Physical variables for different training tests.

Table (2)

ANOVA for Grip Strength.

\begin{tabular}{|c|c|c|c|c|c|}
\hline Grip Strength & Sum of Squares & Df. & Mean Square & F & Sig. \\
\hline Between Groups & 17.607 & 2 & 8.804 & 40.884 & .000 \\
\hline Within Groups & 5.781 & 30 & 0.324 & & \\
\hline Total & 23.388 & 32 & & & \\
\hline
\end{tabular}

Table (3)

ANOVA for VO2 Max.

\begin{tabular}{|c|c|c|c|c|c|}
\hline VO2 Max & Sum of Squares & Df. & Mean Square & F & Sig. \\
\hline Between Groups & 14.184 & 2 & 7.092 & 29.343 & .000 \\
\hline Within Groups & 6.382 & 30 & 0.210 & & \\
\hline Total & 20.566 & 32 & & & \\
\hline
\end{tabular}

Table (4)

ANOVA for LS.

\begin{tabular}{|c|c|c|c|c|c|}
\hline LS & Sum of Squares & Df. & Mean Square & F & Sig. \\
\hline Between Groups & 512.651 & 2 & 256.326 & 23.168 & .000 \\
\hline Within Groups & 263.505 & 30 & 12.056 & & \\
\hline Total & 776.156 & 32 & & & \\
\hline
\end{tabular}

Table (5)

ANOVA for $B S$.

\begin{tabular}{|c|c|c|c|c|c|}
\hline BS & Sum of Squares & Df. & Mean Square & F & Sig. \\
\hline Between Groups & 777.760 & 2 & 388.880 & & .000 \\
\hline Within Groups & 371.000 & 30 & 17.145 & & \\
\hline Total & 1148.76 & 32 & & & \\
\hline
\end{tabular}

Table (6)

ANOVA for SLJ.

\begin{tabular}{|c|c|c|c|c|c|}
\hline SLJ & Sum of Squares & Df. & Mean Square & F & Sig. \\
\hline Between Groups & 1133.569 & 2 & 566.785 & 27.561 & .000 \\
\hline Within Groups & 471.541 & 30 & 23.611 & & \\
\hline Total & 1605.11 & 32 & & & \\
\hline
\end{tabular}

Table (7)

ANOVA for PUT.

\begin{tabular}{|c|c|c|c|c|c|}
\hline PUT & Sum of Squares & Df. & Mean Square & F & Sig. \\
\hline Between Groups & 110.463 & 2 & 55.231 & 11.901 & .000 \\
\hline Within Groups & 120.341 & 30 & 5.526 & & \\
\hline Total & 230.804 & 32 & & & \\
\hline
\end{tabular}


Table (8)

ANOVA for WST

\begin{tabular}{|c|c|c|c|c|c|}
\hline Grip Strength & Sum of Squares & Df. & Mean Square & F & Sig. \\
\hline Between Groups & 743.403 & 2 & 371.701 & 22.932 & .000 \\
\hline Within Groups & 415.611 & 30 & 0.324 & & \\
\hline Total & 1159.014 & 32 & & & \\
\hline
\end{tabular}

The results revealed that significant differences in Grip strength between Strength group and concurrent group for concurrent group. Taking into consideration that significant difference in Grip strength between endurance group and concurrent group for concurrent group, where significant differences in $\mathrm{VO} 2$ max between endurance groups for concurrent group are calculated. There was a significant difference in LS between strength group and endurance group for strength group. No significant differences in LS between strength group and concurrent group were calculated and significant difference in LS between endurance group and concurrent group for concurrent group were calculated as well. While significant differences in BS between strength group and endurance group for strength group were calculated. No significant differences in BS between endurance group and concurrent group, and significant differences in BS between endurance group and concurrent group for concurrent group were calculated. In conclusion, the study has shown that eight weeks of concurrent strength and endurance training has beneficial effects on musculoskeletal power and VO2 max.

\section{Discussion}

The main objective of the study was concerned to determine if concurrent training can enhance VO2, LS, BS, SLJ, WST, PUT, among young tennis players. The main findings of the study were the significant improvements in the physical variables, and VO2 Max, which proved the concurrent training efficacy.

More recent researches concurred with the findings of Ellenbecker et al. (2003). They indicated that agility was the only physical performance variable used to predict competitive rankingsin 83 prepubescent tennis players.Investigating relationships among an array of performanceand clinical variables (e.g., dynamic, isometric, and isokineticstrength; joint laxity and flexibility; speed; agility; peakoxygen consumption; ball velocities of the serve, forehandand backhand) in female collegiate (20 years old) tennisplayers, Kraemer et al. (1995) observed that no single variablestrongly explained tennis performance. Taken together, thesefindings also reinforce that performance in tennis is multifactorial;it depends on mental, tactical, and technical factors,as tennis stroke ratings appeared to be strong predictors oftournament play in preadolescent tennis players.

Hakkinen, et al. (2005) performed a study showing that participants who had resistance trained showed greater improvements in short and long-term endurance compared to those who only endurance trained. Short-term endurance was 5-8 min to exhaustion and long term was maximal cycling time to exhaustion at $80 \%$ VO2 Max. It was hypothesized that resistance training increased short-term endurance performance by increasing highenergy phosphate and glycogen stores. Shortterm endurance may have also been improved by increase in the fast twitch to slow twitch fiber area ratio.

\section{Recommendations}

On the light of the findings from the conclusions, the researcher can state some of recommendations as follows:

1. Outsourcing the training program to be applied on the tennis young players due to what evidenced by the results of this study of improvements.

2. Continue providing training programs with different laboratory measurements.

3. Holding training courses for coaches to educate and training them.

4. The importance of using this training program of the study resulted in the treatment of the weaknesses arising tennis players.

5. The need for further studies in this area to different training loads because of their importance in the field of sports training. 
6. This study can be applied on the different samples and other sports activities.

\section{References}

1. Balabinis, C. P., Psarakis, H., Charalampos, M., Moukas, M.P., Vassiliou, P.K. (2003) 'Early Phase Changes by Concurrent Endurance and Strength Training', J Strength and Conditioning Research 17(2): 393-401.

2. Bell, G.J.,Syrotuik, D., Martin, T.P., Burnham, R. \&Quinney, H.A. (2000), 'Effect of concurrent strength and endurance training on skeletal muscle properties and hormone concentrations in human', European journal of applied physiology, vol.81, no. 5, pp.418-427.

3. Bencke, J, Damsgaard, R, Saekmose, A, Jorgensen, $\mathrm{P}$, Jorgensen $\mathrm{K}$, and Klausen, $\mathrm{K}$. Anaerobic power and muscle strength characteristics of 11 year old elite and non-elite boys and girls from gymnastics, team handball, tennis and swimming. Scand J Med Sports 12: 171-178, 2002.

4. Brooks, G.A. (2000) 'Exercise Physiology: Human Bioenergetics and Its Applications', Mountain View, CA: Mayfield Publishing Company, pp.401-419, 669.

5. Crameri, RM., Aagaard, P., Qvortrup, K., Langberg. H., et al. (2007) 'Myofiberdamage in human skeletal muscle: effects of electrical stimulation versus voluntary contractions', J Physiol, 583:365-80

6. Chelly, SM, and Denis, C. Leg power and hopping stiffness: relationship with sprint running performance. Med Sci Sports Exerc 33: 326-333, 2001.

7. Dantas, EHM, Viana MV, Cader SA, et al. (2008), 'Effects of a programme for yearsenderers physical force on the muscle and body composition for adults.' Sports Science Health; 4: 15- 19.

8. Dalleau, G, Belli, A, Viale, F, Lacour, JR, and Bourdin, M. A simple method for field measurements of leg stiffness in hopping. Int $\mathbf{J}$ Sports Med 25: 170-176, 2004.

9. Davis, WJ, Wood DT, Andrews RG, et al. (2008) 'Concurrent training enhances athletes strength, muscle endurance, and other measures.' J Strength Cond Res; 22(\%): 1487 1502.

10. Ellenbecker, TS, and Roetert, EP. Isokinetic profile of elbow flexion and extension strength in elite junior tennis players. J Orthop Sports PhysTher 33: 79-84, 2003.

11. Ellenbecker, TS, Roetert, EP, Sueyoshi T, and Riewald, S. A descriptive profile of agespecific knee extension flexion strength in elite junior tennis players. Br J Sports Med 41: 728732, 2007.

12. Ferrauti A., Neumann G, Weber K, Keul J. (2002) 'Urine catecholamine Concentrations and psychological stress in elite tennis under practice and tournament conditions', J Sports Med Phys Fitness; 41: 269-74.

13. Gravelle, B;L., and Blessing, D.L. (2000) 'Physiological Adaption in women ConcurrentlyTraining for Strength and Endurance'. J Strength and Conditioning Research 14: 5-13.

14. Girard, O, Micallef, JP, and Millet, GP. Lower-limb activity during the power serve in tennis: effects of performance level. Med Sci Sports Exerc 37: 1021-1029, 2005.

15. Hakkinen, A., Pakarinen, A., Hannoneen, P., Kautiainen, H., Nyman, K., Kraemer W.J., and Hakkinen, K.(2005) 'Effects of prolonged combines strength and endurance training on physical fitness, body composition and serum hormones in women with rheumatoid arthritis and in healthy controls', Clinical and experimental rheumatology, vol.23, no.4, pp. 505-512.

16. Izquierdo, M., Hakkinen K., Ibanez, J, et al. (2005) 'Effects of combined resistance and cardiovascular training on strength, power, muscle cross-sectional area and endurance markers in middle-aged men'. Eur $\mathbf{J}$ ApplPhysiol; 94:70-75.

17. Jung, AP. (2003) 'The impact of resistance training on distance running performance', Sports Med.; 33(7): 539-552.

18. Kadi, F., and Thornell, L.E., (2000) 'Concomitant increases in myonuclear and Satellite cell content in female trapezius muscle following strength training', Histochem, Cell Biol, 113: 99 -103. 
19. McCarthy, JP.,Pozniak, MA., Agre, J. 21. Nieman, D.C (2003) 'Exercise Testing and (2002) 'Neuromuscular adaption to concurrent Prescription: A health-related approach'. New strength and endurance training. Med Sci Sports York: McGraw-Hill, pp. 79 -110.

Exerc; 34(3): 511-519.

22. Philippaerts, RM, Vaeyens, R, Janssens, M, 20. Malina, RM, Cumming, SP, Kontos, AP, Van renterghem, B, Matthys, D, Craen, R, Eisenmann, JC, Ribeiro, B, andAroso, J. Bourgois, J, Vrijens, J, Beunen, G, and Malina, Maturity-associated variation in sport-specific RM. The relationship between peak height skills of youth soccer players aged 13-15 years. velocity and physical performance in youth J Sports Sci 23: 515-522, 2005. soccer players. J Sports Sci 24: 221-230, 2006. 